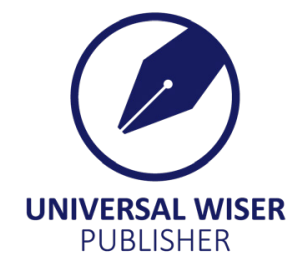

\title{
Visible Light Induced Photocatalytic Dye Degradation by Cobalt Oxide Nanoparticles
}

\author{
Biswajit Chowdhury ${ }^{1}$, Sitangshu Shekhar Pradhan ${ }^{2}$, Hari Sankar Das ${ }^{1}$, Bhaskar Biswas ${ }^{1 *}$ \\ ${ }^{1}$ Department of Chemistry, University of North Bengal, Darjeeling 734013, India \\ ${ }^{2}$ Department of Physics, Midnapore College (Autonomous), Midnapore 721101, India \\ E-mail: bhaskarbiswas@nbu.ac.in,icbbiswas@gmail.com
}

\begin{abstract}
Considering the impact of organic dyes as industrial effluents on the environment, this research study describes the synthesis, structural and morphological characterization, and visible light induced photo-catalytic studies of cobalt oxide nanoparticles (CoONPs). In order to synthesize the CoONPs, a tetra-nuclear cobalt(III)-Schiff base complex was prepared in crystalline phase and employed to produce CoONPs through thermal decomposition of the synthetic precursor. The morphology of the nanoparticle has been delineated with different spectroscopic and analytic methods. Scanning and transmission electron microscopy (FE-SEM and HR-TEM) analyses estimate the cauliflower structured CoONPs with an average size $\sim 80 \mathrm{~nm}$. The photo-catalytic behavior of CoONPs has been tested against murexide (MX) and titan yellow (TY) dye in aqueous phase. This CoONPs ensure more than $90 \%$ of degradation for MX while $\sim 60 \%$ degradation has been observed for TY in aqueous phase for a same time interval. MX and TY adopt different structures which bring the variation in rate of photosensitization for the synthetic CoONPs photocatalyst and account on the different photocatalytic efficiency towards the degradation of MX and TY in presence of visible light.
\end{abstract}

Keywords: cobalt oxide nanoparticles, electron microscopy images, photocatalyst, photo-catalytic behaviour, dye degradation

\section{Introduction}

Nano-structured materials have been considered as an emergent class of functional materials for their unique molecular properties as well as their momentous contributions in designing smart devices of modern use ${ }^{[1-5]}$. Scientific studies on nano-structured materials suggest that variation in morphology may add novel characteristics to magnetic behaviours, catalytic activities, electronic devices, drug design and others ${ }^{[6-16]}$. Among the diversified nano-structured materials, cobalt oxide nanoparticles a $p$-type semiconductor material has been developed extensively due to its various applications in developing nano-magnets ${ }^{[7,8]}$, nano-scale photo-catalysts as well as electro-catalysts ${ }^{[9-12]}$, nano-magnetic sensors ${ }^{[13,14]}$, semiconductors and imaging devices ${ }^{[15]}$, super capacitors and batteries ${ }^{[16,17]}$, improvement of coating materials ${ }^{[18]}$ and others. Noteworthy, nano-scale iron oxides are found as chemically and thermally stable materials and also widely apply in applications of energy conversion ${ }^{[9,19]}$.

The semiconductor-based photocatalysis towards the degradation of toxic industrial dyes has been attracting much attention in recent years. In this process, the semiconductor-based photocatalyst absorbs a photon of energy greater than or equal to the band-gap energy $\left(\mathrm{E}_{\mathrm{g}}\right)$ of the semiconductor and undergoes an electron transfer from the valence band (VB) to the conduction band (CB). As a result of this, an electron-hole pair is generated ${ }^{[20,21]}$. The photo-generated electronhole pairs then react with the adsorbed molecules (e.g., water, oxygen, hydroxyl, etc.) on the surface/interface of the photocatalyst, ultimately the hydroxyl radicals $(\bullet \mathrm{OH})$ are generated, which are highly oxidative in nature $\left(\mathrm{E}^{0}\right.$ $=+2.80 \mathrm{~V})^{[22]}$ and oxidizes organic pollutants into simpler/nontoxic substances. Several metal oxides semiconductors such as $\mathrm{TiO}_{2}, \mathrm{ZnO}, \mathrm{Fe}_{2} \mathrm{O}_{3}, \mathrm{CdS}, \mathrm{ZnS}$ and $\mathrm{V}_{2} \mathrm{O}_{5}$ can act as photocatalysts but $\mathrm{TiO}_{2}$ and $\mathrm{ZnO}$ have been widely explored as photocatalysts for various dyes due to their ability to break down organic pollutants and even achieve complete mineralization ${ }^{[23-26]}$. The main drawback of these materials is the large band gap (3.0-3.2 eV) which limits its efficiency to work only under UV irradiation and poor performance under visible light irradiation ${ }^{[23,24,27]}$. To improve the response of these photocatalyst in presence of visible light, several methods such as surface modification via organic materials and semiconductor coupling, band gap modification by creating oxygen vacancies and oxygen sub-stoichiometry, by metal and nonmetal doping etc ${ }^{[25,26]}$. The scientists are also tried to combine two or more semiconductor metal oxides having

Copyright (C2020 Bhaskar Biswas, et al.

DOI: https://doi.org/10.37256/fce.122020559

This is an open-access article distributed under a CC BY license

(Creative Commons Attribution 4.0 International License)

https://creativecommons.org/licenses/by/4.0/ 
different band gaps for the visible light driven photocatalytic degradation of organic dyes ${ }^{\text {[28] }}$. Spinel-based materials can be used as an alternative candidate because of their band gap values always lies within the range (1.7-2.4 eV) and matches with visible light absorption. Tricobalt tetraoxide nano particles (CoONPs) have the regular spinel structure are considered as a promising material for selective and efficient degradation of industrial organic pollutants even under the visible light irradiation. It is also reviewed that CoONPs were mostly studied for methylene blue dye only ${ }^{[29]}$. In 2015 , C. Ravi Dhas et al. ${ }^{[23]}$ were also studied the photocatalytic degradation of Rhodamine B and Direct Red 80 under visible light by using CoONPs synthesized via sol-gel route. To elucidate the use of CoONPs in dye degradation, we have synthesized CoONPs by the thermal decomposition of tetra-cobalt-Schiff base precursor at $500^{\circ} \mathrm{C}$ for $5 \mathrm{~h}$ in a furnace and studied as a photocatalyst for the visible light induced degradation of murexide and titan yellow in aqueous medium.

\section{Experimental}

\subsection{Preparation of the precursor}

\subsubsection{Chemicals, solvents and starting materials}

High purity $O$-vanilin (Aldrich, USA), 1,3-diaminopropan-2-ol (Aldrich, USA) and cobalt(II) nitrate hexahydrate (Merck, India) were purchased from the respective concerns. All the chemicals and solvents were of Analytical grade (A.R. grade) and used as received without further purification.

\subsubsection{Preparation of the Schiff base $\left(\mathrm{H}_{3} \mathrm{~L}\right)$}

The Schiff base, $\mathrm{H}_{3} \mathrm{~L}$ and its tetra-cobalt(III) complex were prepared following a reported literature ${ }^{[30-33]}$. To synthesis $\mathrm{H}_{3} \mathrm{~L}, O$-vanillin $(0.3060 \mathrm{~g}, 2 \mathrm{mmol})$ was mixed with 1,3 -diaminopropan-2-ol $(0.0890 \mathrm{~g}, 1 \mathrm{mmol})$ in $40 \mathrm{ml}$ methanol and kept on magnetic stirrer for $\sim 2 \mathrm{~h}$. By this time, bright yellow crystalline compound was separated out. The Schiff base was filtered off, and stored in vaccuo over $\mathrm{CaCl}_{2}$ for subsequent use. Yield, $0.2760 \mathrm{~g}(82.60 \%)$. Anal cal. for $\mathrm{C}_{17} \mathrm{H}_{18} \mathrm{~N}_{2} \mathrm{O}_{3}\left(\mathrm{H}_{3} \mathrm{~L}\right)$ : C, 68.48; H, 6.08; N, 9.39; Found: C, 68.40; H, 6.02; N, 9.35. IR (KBr, cm $\left.{ }^{-1}\right): 1631$ (s), 3378 (s); UV-Vis $\left(\lambda_{\max }, \mathrm{nm}\right): \sim 220$, $255,317,405 \mathrm{~nm}$.

\subsubsection{Preparation of the tetra-cobalt(III)-Schiff base complex (1)}

Crystalline $\mathrm{Co}\left(\mathrm{NO}_{3}\right)_{2} \cdot 6 \mathrm{H}_{2} \mathrm{O}(0.580 \mathrm{~g}, 2 \mathrm{mmol})$ was added portionwise to $\mathrm{H}_{3} \mathrm{~L}(0.360 \mathrm{~g}, 1 \mathrm{mmol})$ in methanol and kept the solution on a magnetic stirrer for 30 mins. The yellow solution of the ligand turned dark brown. The reaction mixture was kept in air for slow evaporation to obtain pure crystalline compounds. After a week, reddish-brown coloured fine crystalline compound was separated out from the reaction mixture. The crystalline product was washed with toluene and dried in vacuum over silica gel indicator.

Yield of 1: $0.360 \mathrm{~g}\left(62.0 \%\right.$ based on metal salt). Anal. Calc. for $\mathrm{C}_{44} \mathrm{H}_{60} \mathrm{~N}_{4} \mathrm{O}_{16} \mathrm{Co}_{4}$ (1): C, 46.49; H, 5.32; N, 4.93. Found: C, 46.89; H, 5.37; N, 4.98\%. IR (KBr pellet, $\left.\mathrm{cm}^{-1}\right): 3455\left(v_{\mathrm{OH}}\right), 1637,1611\left(v_{\mathrm{C}=\mathrm{N}}\right)$; ESI-MS (m/z) 1138.87.

\subsubsection{Preparation of the cobalt oxide nanoparticles (CoONPs)}

The cobalt oxide nanoparticles (CoONPs) were synthesized by thermal decomposition of this tetra-cobalt cluster as a precursor. Temperature variable decomposition plot (Figure 1) of cobalt(III)-Schiff cluster displayed stepwise decomposition. Increasing the heating with progress of time led the production of CoONPs. In this case, this cobalt cluster was heated for $5 \mathrm{~h}$ at $500^{\circ} \mathrm{C}$ in a muffle furnace and the resultant thermally stable end product was obtained as CoONPs. Different spectroscopic methods and analytical techniques were employed to characterize CoONPs. 


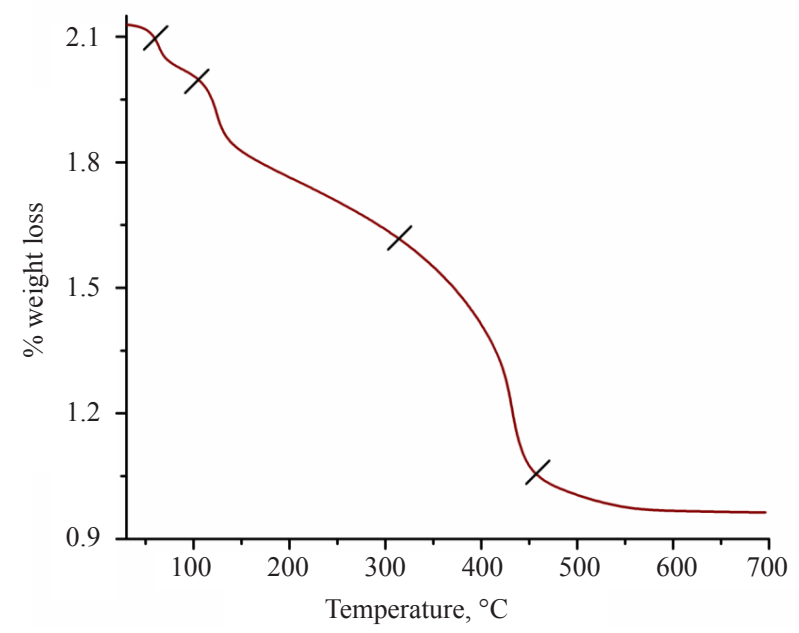

Figure 1. Thermogravimmetric analysis spectrum of the tetracobalt-Schiff base complex

\subsection{Physical measurements}

A spectrophotometer (FTIR-8400S SHIMADZU) was employed to record infrared spectrum (KBr) of tetracobalt cluster and CoONPs in the range 400-3600 $\mathrm{cm}^{-1} .{ }^{1} \mathrm{H}$ and ${ }^{13} \mathrm{C}$ NMR spectra were obtained on a Bruker Avance $300 \mathrm{MHz}$ spectrometer in DMSO- $d_{6}$ at $25^{\circ} \mathrm{C}$. Ground state absorption was measured with a JASCO V-730 UV-Vis spectrophotometer. Thermal analysis was carried out on a PerkinElmer Diamond TG/DTA system up to $800^{\circ} \mathrm{C}$ in a static nitrogen atmosphere with a heating rate of $10^{\circ} \mathrm{C} / \mathrm{min}$. Elemental analyses were performed on a Perkin Elmer $2400 \mathrm{CHN}$ microanalyser.

\subsection{Crystal structure determination and refinement}

Single crystal X-ray diffraction data are collected using Bruker and Rigaku XtaLABmini diffractometer equipped with Mercury 375R $(2 \times 2$ bin mode $)$ CCD detector. We had collected three different set of data for this tetra-cobaltSchiff base cluster using three different batches of crystals; however for the poor quality of crystals we couldn't produce satisfactory refinement parameter to get perfect X-ray structure. The data are collected with graphite monochromated Mo$\mathrm{K} \alpha$ radiation $(\lambda=0.71073 \AA)$ at $100.0(2) \mathrm{K}$ using $\omega$ scans. The data are reduced using Crystal Clear suite, and the space group determination is done using OLEX ${ }^{2}$. The structure is resolved by direct method and refined by full-matrix leastsquares procedures using the SHELXL-97 software package using OLEX ${ }^{2}$ suite ${ }^{[34-37]}$.

\subsection{Physicochemical characterization of cobalt oxide nanopartcles}

The morphology and structure of cobalt oxide nanoparticles (CoONPs) were assessed through scanning electron microscopy (FE-SEM, HR-TEM) images including energy dispersive X-ray analysis (EDX). The SEM images were recorded on a microscope in JSM-6700F FESEM, JEOL, Japan and TEM images were obtained on a JEM-2100 HR-TEM, JEOL, Japan.

\subsection{Photocatalytic experiments}

The photocatalytic activities of CoONPs were evaluated by degradation of murexide (MX) and titan yellow (TY) dye solutions. All the experiments were carried out in presence of visible light using a previously reported procedure by our group ${ }^{[2,26]}$. A $250 \mathrm{ml}$ Borosil beaker with outside water circulation was placed on a magnetic stirrer, above which a high pressure mercury vapour lamp (100 W, Philips) emitting visible light was placed. CoONPs at a dose of 0.0020g (solid) was added to $100 \mathrm{ml}$ TY and MX dye solutions $\left(1.0 \times 10^{-4} \mathrm{M}\right)$ in separate beakers. The distance of the light source from the upper level of dye solution was $18 \mathrm{~cm}$ for maximum use of light radiation. The solutions were stirred in dark condition for 5 minutes to establish the adsorption equilibrium. The zero time reading was taken on a spectrophotometer and the solutions were irradiated with visible light. Aliquots of $5 \mathrm{ml} \mathrm{CoONPs} \mathrm{were} \mathrm{taken} \mathrm{at} \mathrm{regular} \mathrm{time} \mathrm{interval} \mathrm{(} 8$ minutes) and centrifuged to analyse the percent degradation of the individual dyes. The percentage dye degradation was calculated using formula:

$\%$ Degradation $=\left[\left(A_{0}-A_{t}\right) / A_{o}\right] \times 100$, where, $A_{o}$ is the initial dye absorbance; $A_{t}$ is the dye absorbance at time $(t)$ after treatment with CoONPs. 


\section{Results and discussion}

\subsection{Synthesis of the tetra-cobalt cluster and cobalt oxide nanoparticle (CoONPs)}

The single crystals of tetra-cobalt(III) complex was prepared by mixing hydrated cobalt(II) nitrite with Schiff base in 2:1 molar ratio in methanol. Methanol-acetonitrile solvent mixture is also useful to produce the tetra-cobalt cluster.

The cobalt oxide nanoparticles (CoONPs) were synthesized by thermal decomposition of the tetra-cobalt-Schiff base precursor at $500^{\circ} \mathrm{C}$ for $5 \mathrm{~h}$ in a furnace. The tetra-cobalt cluster was thermally cleaved in a stepwise manner under $\mathrm{N}_{2}$ atmosphere to produce cobalt oxide nanoparticles (Figure 1). The tetra-cobalt-Schiff base precursor yields cobalt oxide nanoparticle in high efficiency as a thermally stable product. The purity of these synthetic nanoparticles was confirmed by energy dispersive X-ray spectrum (EDX) which suggests $\sim 95 \%$ purity of the nanoparticles. The composition of cobalt oxide nanoparticles was determined through different spectroscopic methods. The schematic presentation of the synthetic route of cobalt oxide nanoparticle using thermal decomposition technique is presented below (Figure 2):

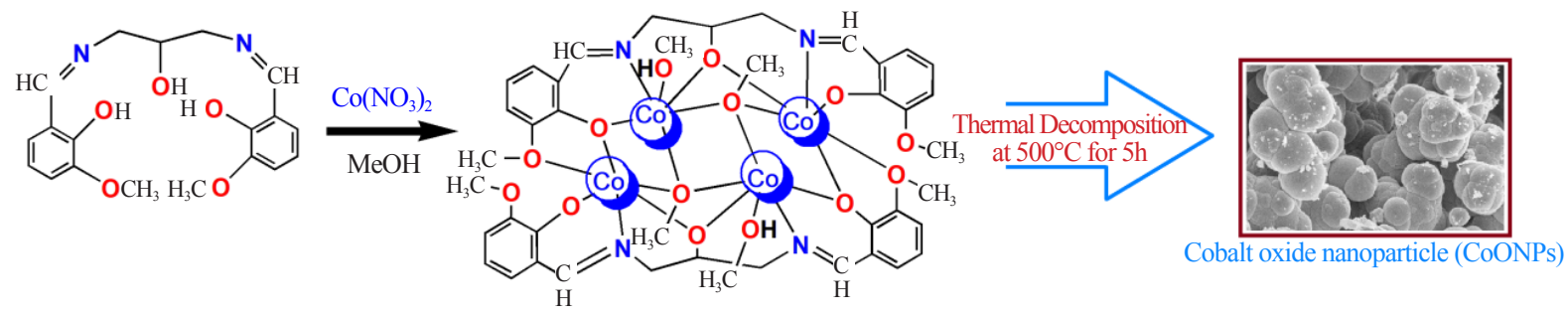

Figure 2. Preparative procedure of the tetra-cobalt(III) precursor and CoONPs

\subsection{Description of crystal structure}

X-ray structure analysis reveals that tetra-cobalt-Schiff base cluster crystallizes in an orthorhombic system with $P$ ben space group. The thermal ellipsoidal plot for this tetra-cobalt-Schiff base cluster is displayed in Figure 3 . The structural refinement data of tetra-cobalt-Schiff base cluster are enlisted in Table 1. Selected bond angles and bond distances of 1 are given in Table 1. The X-ray crystal structure of the cobalt(II)-Schiff base complex (Figure 3) actually exists as a neutral tetra metallic core, $\left[\mathrm{Co}_{4}(\mathrm{~L})_{2}\left(\mu_{3}-\mathrm{OCH}_{3}\right)_{2}\left(\mathrm{CH}_{3} \mathrm{OH}\right)_{2}\right]$. The tetra-cobalt cluster was formed by four cobalt ions in combination with two Schiff base and two methoxide ions. Very interestingly, methanol has played a significant role in the formation of cluster. Methanol acts as a terminal ligand and a bridging ligand and presents its unique coordination motifs in this tetra-metallic core. All the cobalt centres adopt distorted octahedral geometry (Figure 3) and two [Co $(\mathrm{L})]$ units are interconnected to each other through two methoxide ions. Each of the cobalt centres adopts an octahedral geometry. The quality of crystals was not good and thereby, crystallographic refinement parameters were not satisfactory which is also evident from its high $\mathrm{R}$ factor. However, X-ray diffraction study provides sufficient information about the structural features and composition of the tetra-metallic cobalt complex. Literature survey on structurally similar tetranuclear cobalt-Schiff base complex indicates that L. Xiang et al. previously reported the synthesis and magnetic aspects of a tetracobalt(II)-Schiff base with the same Schiff base using different reaction methodology ${ }^{[38]}$. This tetra-cobalt-Schiff base complex has got the identical crystal structure to the previously reported cobalt cluster. We recorded three sets of crystallographic data for our synthesized cobalt-Schiff base compound, although each and every times we found R-factor very high. The details of structural description and coordination linkages of this tetracobalt-Schiff base cluster are described by L. Xiang et al. elsewhere ${ }^{[38]}$. 


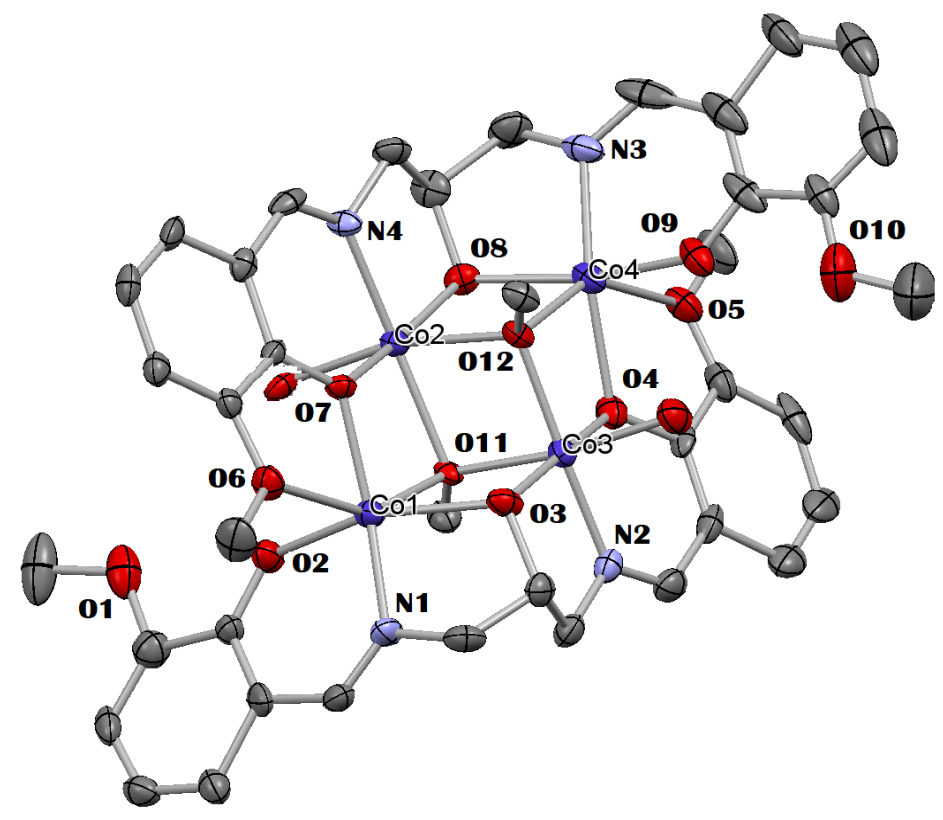

Figure 3. X-ray structure of tetra-cobalt-Schiff base

Table 1. Comparative literatures on photocatalytic degradation of different dyes using CoONPs

\begin{tabular}{|c|c|c|c|c|c|}
\hline Photocatalyst & Dyes & $\begin{array}{l}\text { Photosource/catalyst } \\
\text { conc. }\left(\mathrm{gL}^{-1}\right)\end{array}$ & $\begin{array}{l}\text { Time } \\
(\mathrm{min})\end{array}$ & $\%$ degradation efficiency & References \\
\hline $\mathrm{Co}_{3} \mathrm{O}_{4} \mathrm{NPs}$ & Rhodamine & Vis $\left(0.3 \mathrm{gL}^{-1}\right)$ & 30 & 32 & $20 \mathrm{a}$ \\
\hline $\mathrm{Co}_{3} \mathrm{O}_{4} \mathrm{NPs}$ & Direct Red 80 & Vis $\left(0.3 \mathrm{gL}^{-1}\right)$ & 30 & 78 & $20 \mathrm{a}$ \\
\hline $\mathrm{Co}_{3} \mathrm{O}_{4} \mathrm{NPs}$ & Methylen blue & UV-Vis & 420 & 55.71 & 31 \\
\hline $\mathrm{Cr}$ doped $\mathrm{Co}_{3} \mathrm{O}_{4} \mathrm{NPs}$ & Methylene blue & Vis $\left(2.0 \mathrm{gL}^{-1}\right)$ & 35 & 99 & 32 \\
\hline $\mathrm{Co}_{3} \mathrm{O}_{4} \mathrm{NPs}$ & EBT & Solar light $\left(0.1 \mathrm{gL}^{-1}\right)$ & 30 & 39.4 & 33 \\
\hline $\mathrm{Co}_{3} \mathrm{O}_{4} \mathrm{NPs}$ & Murexide & Solar light $\left(0.25 \mathrm{gL}^{-1}\right)$ & 40 & 43.6 & 33 \\
\hline Doped $\mathrm{Co}_{3} \mathrm{O}_{4} \mathrm{NPs}$ & Eosin blue & $\mathrm{UV}-\mathrm{Vis}\left(0.8 \mathrm{gL}^{-1}\right)$ & 110 & 95 & 34 \\
\hline $\mathrm{Co}_{2} \mathrm{ZrO}_{5} \mathrm{NPs}$ & Murexide & Vis $\left(0.01 \mathrm{gL}^{-1}\right)$ & 90 & 97 & 36 \\
\hline $\mathrm{Co}_{3} \mathrm{O}_{4} \mathrm{NPs}$ & Murexide & Vis $\left(0.2 \mathrm{gL}^{-1}\right)$ & 40 & 92.2 & This work \\
\hline $\mathrm{Co}_{3} \mathrm{O}_{4} \mathrm{NPs}$ & Titan Yellow & Vis $\left(0.2 \mathrm{gL}^{-1}\right)$ & 40 & 51.4 & This work \\
\hline ZnONPs & Titan Yellow & Vis $\left(1.0 \mathrm{gL}^{-1}\right)$ & 60 & 96 & 37 \\
\hline
\end{tabular}

\subsection{Physico-chemical characterization of CoONPs}

Various spectroscopic and analytical techniques have been used to characterize the morphology and structure of cobalt oxide nanoparticles (CoONPs). IR spectrum of the CoONPs showed a characteristic peak at $561 \mathrm{~cm}^{-1}$ which is assignable to cobalt-oxide (Co-O) stretching of the CoONPs (Figure 4) ${ }^{[24,26]}$. A small but broad peak at $\sim 3550 \mathrm{~cm}^{-1}$ was also appeared in IR spectrum of CoONPs which was probably due to absorption of moisture by CoONPs or KBR. No other peaks were observed which indicates the purity of synthetic CoONPs. 


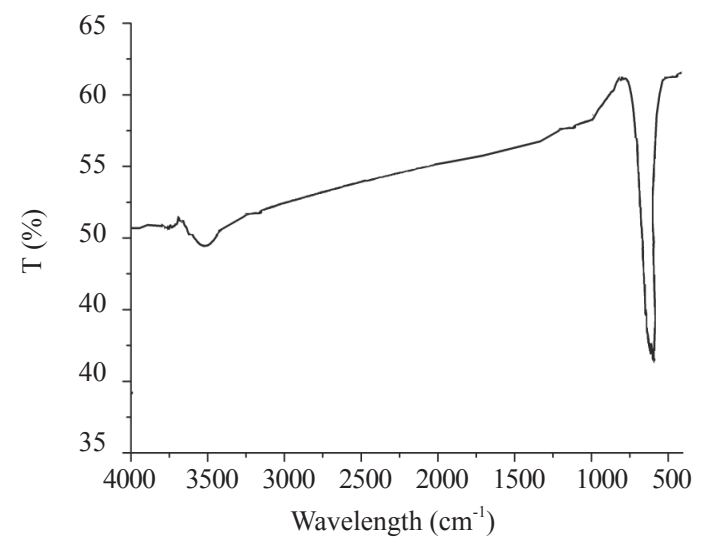

Figure 4. IR spectrum of CoONPs

An electronic spectrum of CoONPs has been measured in the wavelength range of 200 to $600 \mathrm{~nm}$ in aqueous medium (Figure 5). A highly intense electronic band at $\lambda_{\max }=330 \mathrm{~nm}$ with a tail in the visible region and a relatively intense electronic band at $\lambda_{\max }=469 \mathrm{~nm}$ were observed. The characteristic band at $330 \mathrm{~nm}$ is assignable for $\mathrm{n} \rightarrow \sigma^{*}$ electronic transition for the synthetic CoONPs ${ }^{[23,24]}$. The existed electronic bands are consisted with those of the previously reported data ${ }^{[23-26]}$.

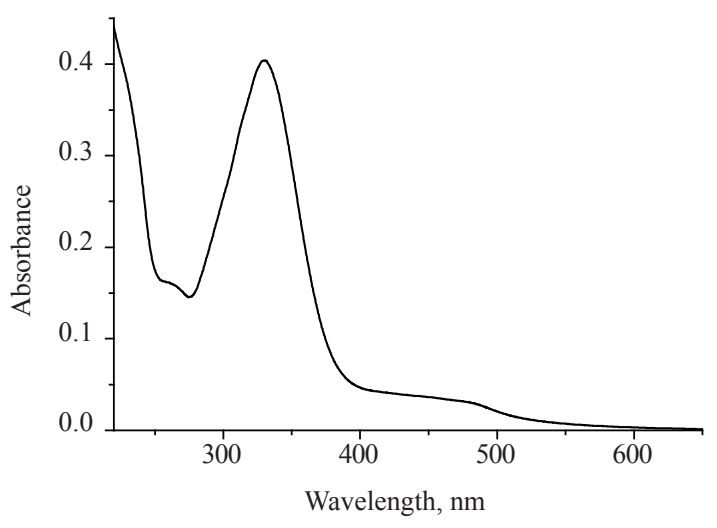

Figure 5. UV-Vis spectrum of CoONPs in water medium

The band gap energy of CoONPs was determined based on the appearance of the electronic bands in the absorption spectrum of CoONPs following the equation, $\mathrm{E}_{\mathrm{bg}}=1240 / \lambda(\mathrm{eV})$, where, $\mathrm{E}_{\mathrm{bg}}$ is the band gap energy of the photocatalyst, $\lambda$ is the wavelength in $\mathrm{nm}$. The band gap energy of CoONPs is calculated as $3.76 \mathrm{eV}$ (Figure 5). This band gap energy of CoONPs is suitable to act as a good photo-catalyst.

The hydrodynamic diameter of CoONPs in aqueous phase was estimated by dynamic light scattering (DLS) study. The DLS profile of CoONPs is shown in Figure 6. The hydrodynamic size measurement suggested that CoONPs had an average size of $63 \mathrm{~nm}$ in aqueous medium. The electrokinetic potential, also known as zeta potential of a nanosuspension is an important parameter which accounts on its physical stability. The electric potential at the interface of the electrical double layer of CoONPs was determined by measurement of zeta potential using DMSO as a dispersant. The value of zeta potential of CoONPs was calculated as $+33.2 \mathrm{mV}$ in DMSO dispersant (Figure 7). Scientific literature shows that the nanoparticles should have a minimum zeta potential of $30 \mathrm{mV}$ in order to get electrostatically stable and for a combined electrostatic as well as steric stabilization, a minimum $20 \mathrm{mV}$ is desirable ${ }^{[39]}$. In our case, the zeta potential value of 33.2 $\mathrm{mV}$ ensures the stability of CoONPs in solution phase ${ }^{[23,24,39]}$. 


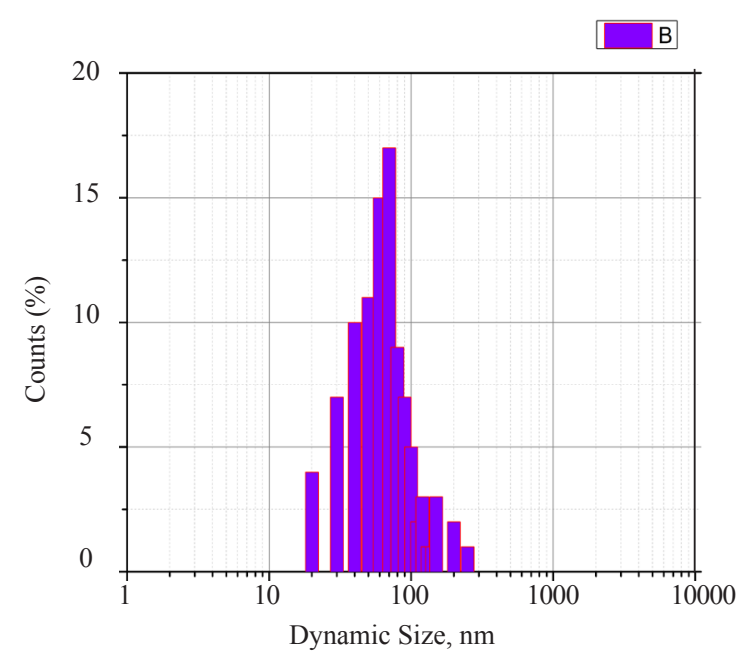

Figure 6. Dynamic scattering light plot of CoONPs

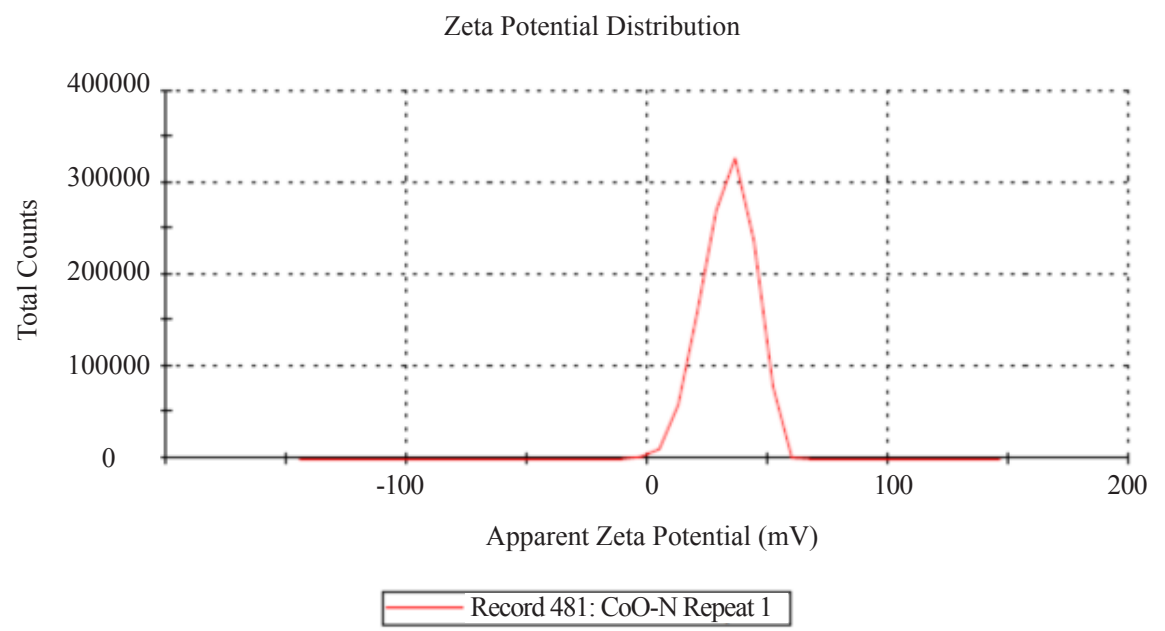

Figure 7. Zeta potential distribution plot of CoONPs

\subsection{Scanning electron microscopy analysis}

The nature of surface morphology and particle size of CoONPs was estimated with FE-SEM and HR-TEM. From the micrographs of SEM (Figure 8), it is revealed that cobalt oxide nanoparticles exist in cauliflower structured morphology with an average particle size $\sim 80 \mathrm{~nm}$. The individual particles of CoONPs exist in an inter-linked porous structure with spherical shape probably. Most of the individual nanoparticles vary from 50 to $100 \mathrm{~nm}$ in size for these synthetic CoONPs. Figure 8 presents the images of pure CoONPs. It can be clearly seen that the individual grain size of the nanoparticles is $\sim 80 \mathrm{~nm}$.

HR-TEM images (Figure 9) were also recorded for better understanding of surface morphology and size of the CoONPs. HR-TEM analysis displayed similar kind of inter-linked surface morphology with irregular shape of individual particle. Close look at surface morphology of CoONPs ensures that individual nanoparticles have well defined surface barrier ranging from 30 to $80 \mathrm{~nm}$ particle sizes. Therefore, scanning electron microscopy image analyses are in well agreement about the morphology of the CoONPs. 

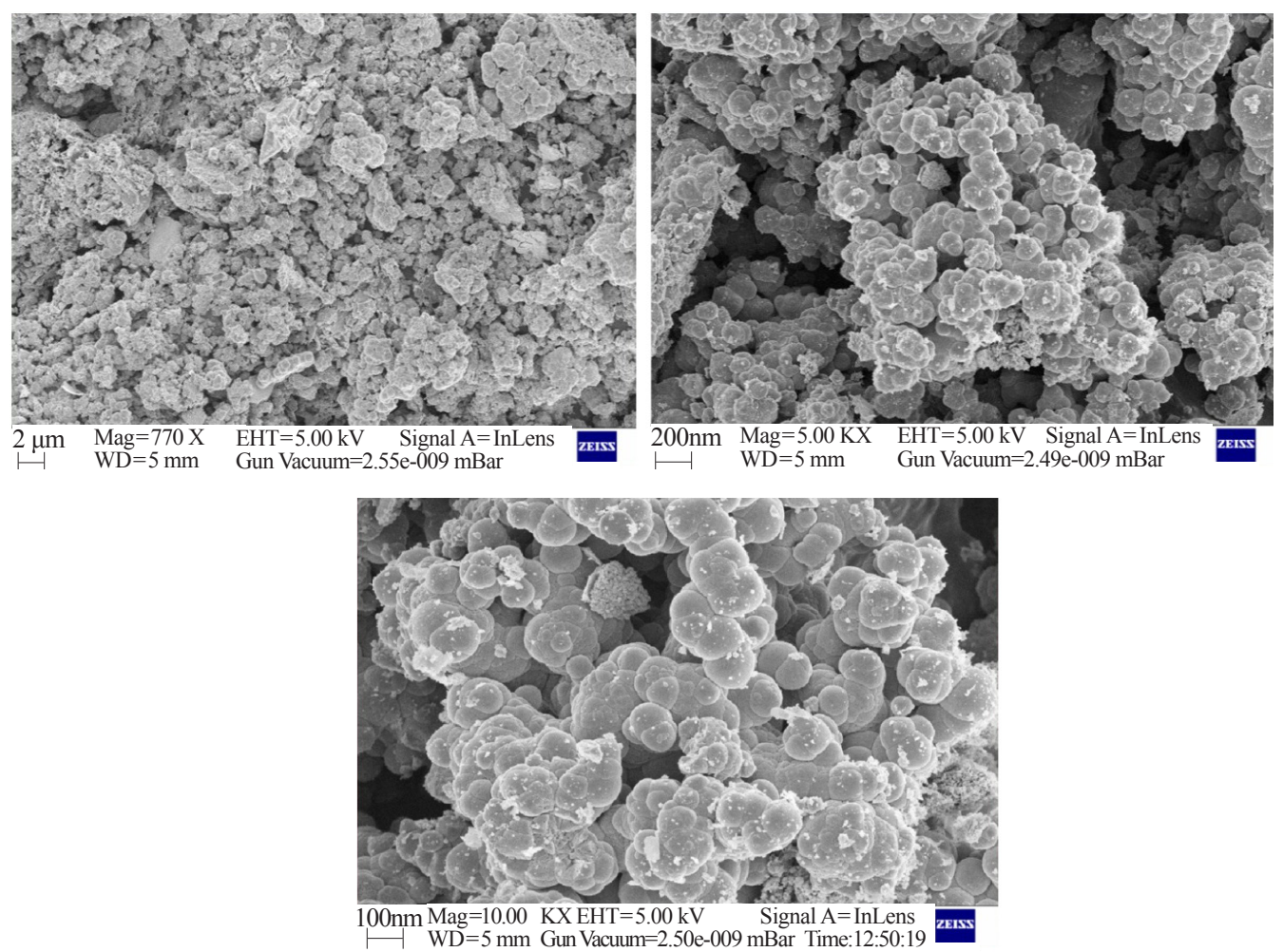

Figure 8. FE-SEM micrographs of CoONPs at different magnification
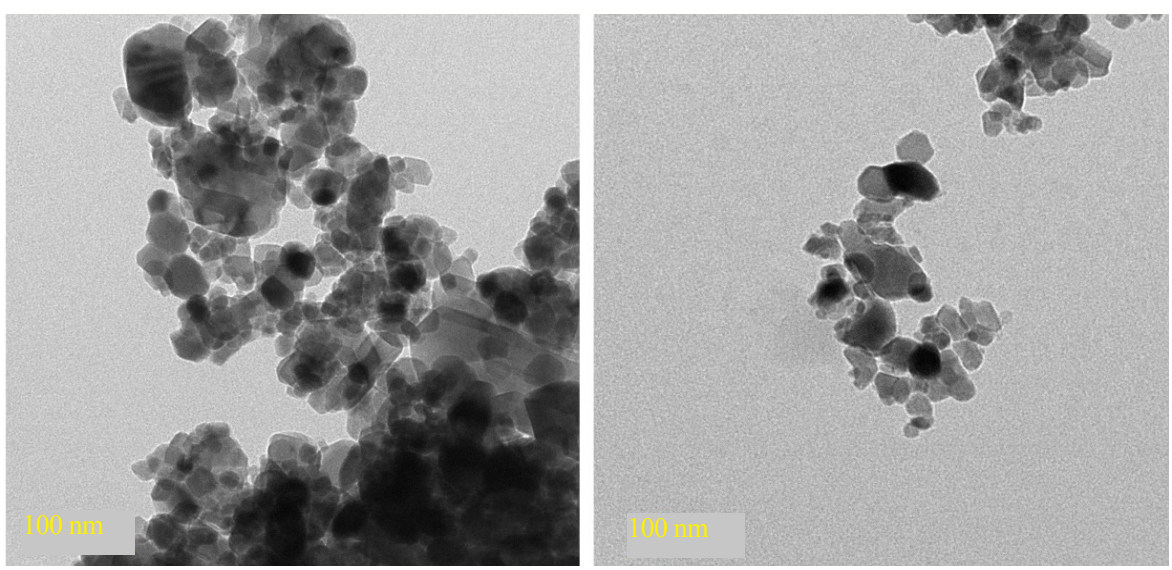

Figure 9. HR-TEM image of CoONPs

\subsection{Photocatalytic degradation study of murexide and titan yellow dyes}

The photocatalytic degradation activities of the synthetic CoONPs towards two different dyes, MX and TY (Figure 10) were studied with $20 \mathrm{mg}$ dose of CoONPs in $100 \mathrm{~mL}$ of aqueous solutions of the dyes under visible light irradiation at $25^{\circ} \mathrm{C}$. The degradation behaviour of the dyes and the kinetics were investigated by time dependent UV-Vis spectroscopy at a 8 min interval for 40 mins. The following Langmuir-Hinshelwood (L-H) expression was applied to estimate the pseudo first order kinetic for the photodegradation of the dye.

$$
\ln \left(C / C_{0}\right)=K_{\mathrm{App}} t
$$

where $k_{\mathrm{App}}$ represents the apparent pseudo first order rate constant of reaction and $\mathrm{t}$ is the reaction time. $\mathrm{C}_{0}$ and $\mathrm{C}$ denote the initial concentration of dye and final concentration of dye at time $t$, respectively. 
The MX, ammonium salt of purpuric (5,5'-nitrilo-dibarbituric) acid (a) is a heterocyclic aliphatic compound shows characteristic long-wave absorption band at $\lambda_{\max }=522 \mathrm{~nm}$ in aqueous medium. Whereas, TY is a heterocyclic aromatic triazene compound shows characteristic long-wave absorption band at $\lambda_{\max }=409 \mathrm{~nm}$ in aqueous medium.

a)<smiles>O=C1NC(=O)C(=Nc2c([18O])[nH]c(=O)[nH]c2=O)C(=O)N1</smiles>

\section{b)}<smiles></smiles>

Figure 10. Chem Draw structure of murexide (MX) and titan yellow (TY)

Upon addition of catalytic amount $(0.0020 \mathrm{~g})$ of synthetic CoONPs to the aqueous solution of MX under visible light exposure, the maximum absorbance of MX (1.007 a.u) at $522 \mathrm{~nm}$ was decreased (Figure 11). When the light was exposed for longer time, larger quantity of MX was degraded with concomitant reduction of the intensity of MX solution. After 40 mins of irradiation of light, most of the MX was degraded (Figure 12) and the degradation efficacy of the synthetic CoONPs were calculated as $92.15 \%$. The catalytic degradation of TY employing same CoONPs was studied under identical reaction conditions and time interval. The reduction of initial absorbance at $409 \mathrm{~nm}$ for TY in aqueous medium was also decrease upon catalytic addition of CoONPs $(20 \mathrm{mg})$ (Figure 13). The visible light was exposed on the reaction medium for a period of 40 mins and the changes of absorbance were monitored with a spectrophotometer. However, in this case, synthetic CoONPs didn't behave as a good photocatalyst as evident from the calculated maximum degradation efficiency $51.0 \%$ at 40 mins (Figure 14). Control experiments were also carried out in absence of CoONPs to testify the effect of synthetic CoONPS on the degradation of dyes under identical reaction conditions. There were minuscule changes of absorbance for both MX and TY dye under visible light exposure which can be neglected. The controlled experiments ensure that synthetic CoONPs remain the driving force for the degradation of MX and TY in aqueous phase. Furthermore, addition of synthetic CoONPs into the aqueous solution of MX and TY in presence of visible light reduced the absorbance of the solution significantly in visible region which is a definite signature of decomposition of the chromophores of MX and TY in aqueous phase. It is further observed that synthetic CoONPs exhibit excellent degradation efficiency towards MX compared to TY.

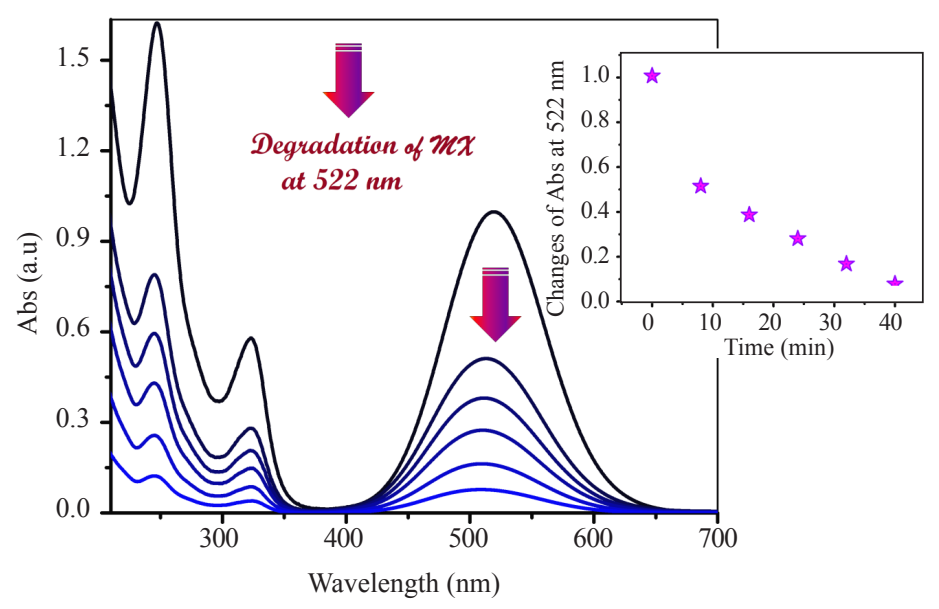

Figure 11. Changes of absorbance of $\mathrm{MX}$ in aqueous solution upon addition of CoONPs under visible light irradiation. Inset: Time vs changes of Abs plot 


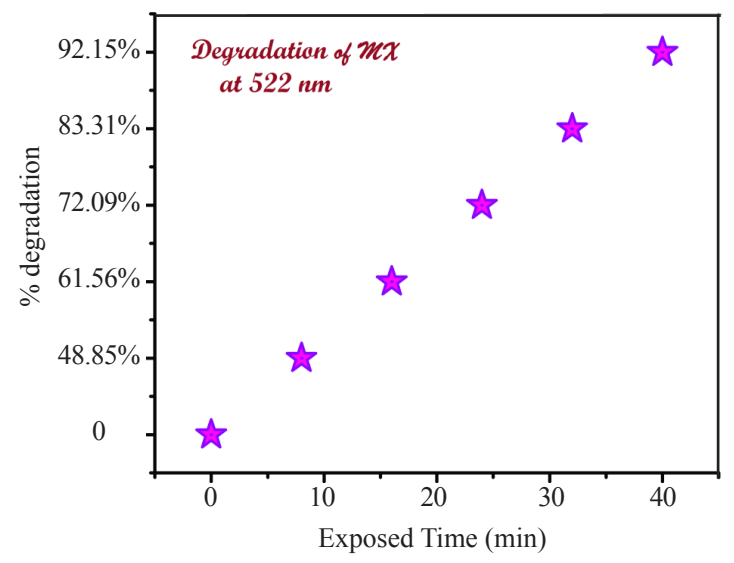

Figure 12. Time dependent photocatalytic activity of CoONPs towards MX in aqueous solution under visible light irradiation

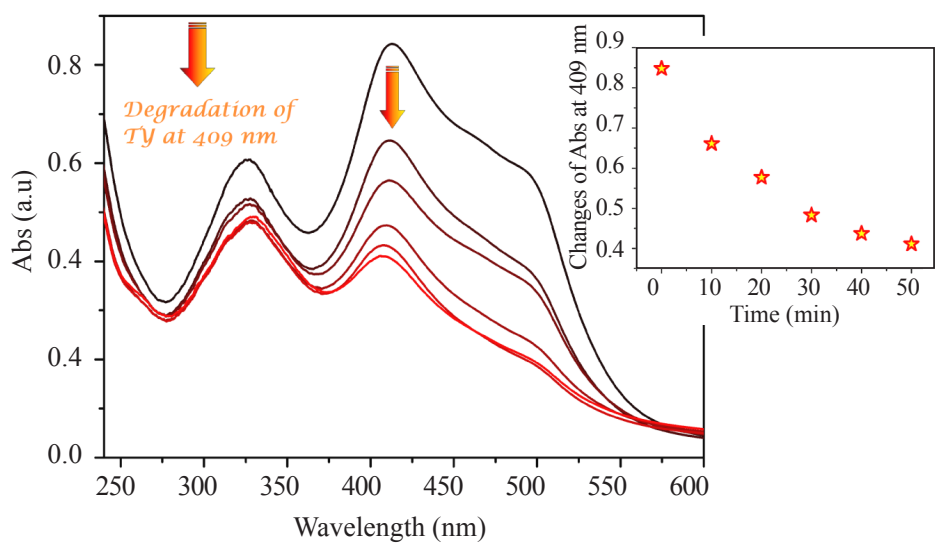

Figure 13. Changes of absorbance of TY in aqueous solution upon addition of CoONPs under visible light exposure. Inset: Time vs changes of Abs plot

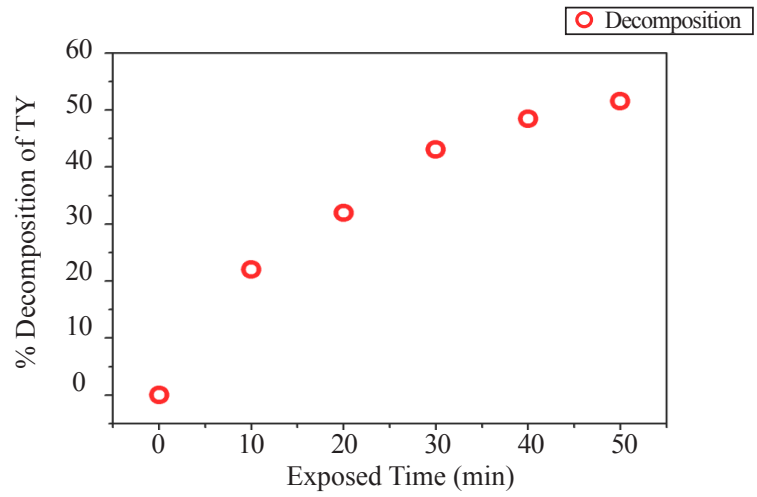

Figure 14. Time dependent photocatalytic activity of CoONPs towards TY in aqueous solution under visible light exposure

We have made a comparison of reactivity among the degradation efficiency of CoONPs photocatalysts towards different dyes and catalysts (Table 1) ${ }^{[40-45]}$. The present CoONPs shows an excellent efficiency compared to the other CoONPs. The photocatalytic efficiency of our synthetic CoONPs was found maximum towards the degradation of MX, while the synthetic CoONPs exhibits moderate activity towards the degradation of TY in aqueous medium. Until date, few photocatalysts are known for the visible light induced degradation MX and TY ${ }^{[33,35,37]}$. For example, Adekunle et al. ${ }^{[41]}$, studied the photocatalytic degradation of MX employing $\mathrm{Co}_{3} \mathrm{O}_{4}$ nanoparticles in aqueous medium and found $\sim 43.60 \%$ degradation efficiency (DE) under solar light irradiation for 40 mins. Recently, Hendekhale et al. ${ }^{[44]}$, have reported the 
highest $\mathrm{DE}$ of $97.0 \%$ at $\mathrm{pH} 5.3$ under visible light irradiation for $90 \mathrm{~min}$ using $\mathrm{Co}_{2} \mathrm{ZrO}_{5}$ NPs. The present $\mathrm{CoONPs}$ shows $92.20 \% \mathrm{DE}$ of $\mathrm{MX}$ at neutral $\mathrm{pH}$ with visible light irradiation indicates faster degradation rate. On the flipside, most of the reported conventional catalysts for the degradation of $\mathrm{TY}$ are $\mathrm{TiO}_{2}$ or $\mathrm{ZnO}$ based nanoparticles induced by UV light ${ }^{[46-48]}$. Recently, Pal et al. ${ }^{[45]}$ have reported an efficient photocatalyst, $\mathrm{ZnO}$ nano particles synthesized by using Moringa Oleifera leaf extract which degrades TY about $96.0 \%$ under 60 min visible light irradiation in the $\mathrm{pH}$ range 2.0-12.0. The present CoONPs photocatalyst degrades TY moderately about $51.40 \%$ in 40 min under visible light irradiation.

\subsection{Photodegradation mechanism}

The semiconductor material based photocatalytic dye degradation mechanisms are well established in the literature ${ }^{[23,24,49,50]}$. The basic mechanism of photocatalytic degradation of dyes is illustrated in Figure 15. The energy from the light excites an electron $\left(e^{-}\right)$from the valence band (VB) to conduction band (CB) of photocatalyst and generates a positive hole $\left(h^{+}\right)$in the VB. The $e^{-}$and $h^{+}$are powerful reducing and oxidizing agents, respectively. Both these entities can migrate to the catalyst surface, where they can enter in a redox reaction with adsorbed molecules (e.g., water, oxygen, hydroxyl, etc.) present on the surface/interface of the catalyst. In most cases $h^{+}$can oxidized the surface bound $\mathrm{H}_{2} \mathrm{O}$ to produce $\bullet \mathrm{OH}$ radicals, whereas, $e^{-}$can reduces the $\mathrm{O}_{2}$ to produce superoxide radical anion $\bullet \mathrm{O}_{2}^{-}$which form $\mathrm{H}_{2} \mathrm{O}_{2}$ by the reaction with two proton $\left(\mathrm{H}^{+}\right)$and one electron which comes from another molecule of $\bullet \mathrm{O}_{2}^{-}$and finally decomposes to $\cdot \mathrm{OH}$ radicals. The $\cdot \mathrm{OH}$ radicals are highly oxidative in nature $\left(\mathrm{E}_{0}=+2.80 \mathrm{~V}\right)^{[22]}$ and oxidizes organic dyes into simpler/ nontoxic substances. Another possible pathway of photoelectron transfer is also reported in the literature by $\mathrm{Fu}$ et al. ${ }^{[51]}$ where dyestuffs can act as photosensitizers shown in Figure 15. In this photochemical process, a light induced electron transfer takes place from the activated dye denoted as dye* (singlet and triplet states) to the molecular oxygen via catalyst and produces radical cation dye $e^{++}$and radical anion $\bullet_{2}^{-}$species and finally, dye ${ }^{+}$get mineralized on reaction with $\bullet \mathrm{O}_{2}^{-} / \mathrm{O}_{2}$.

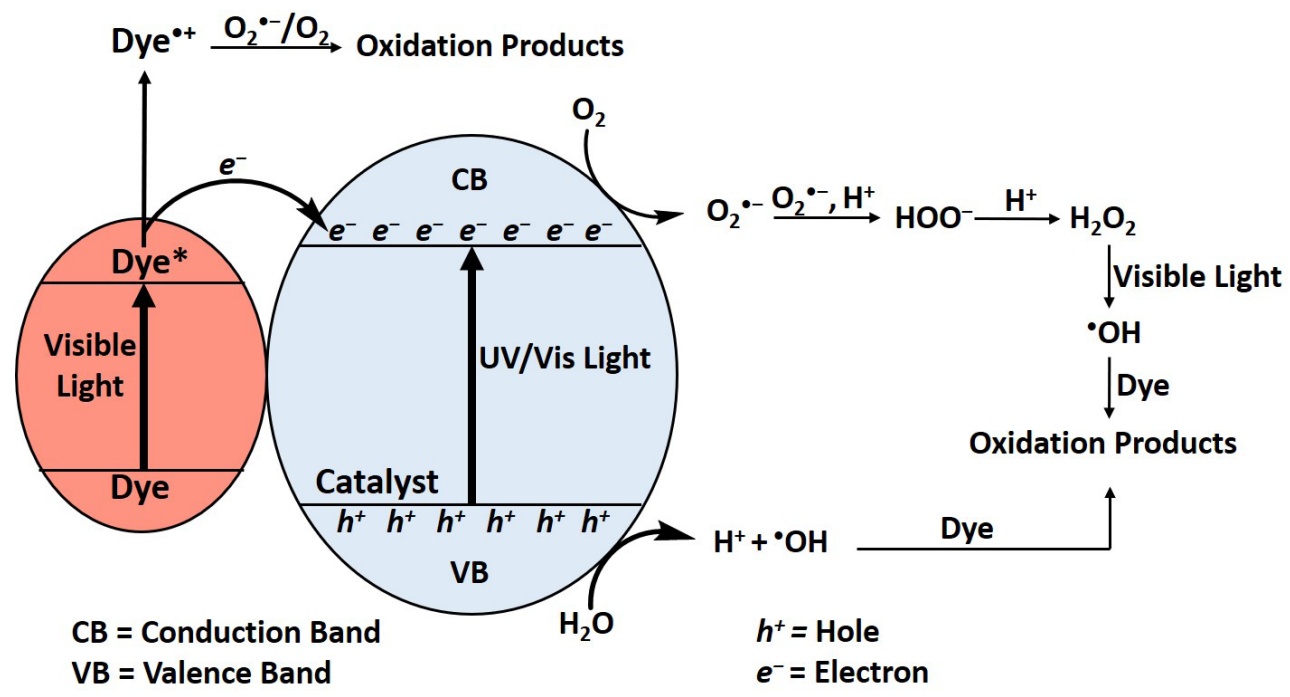

Figure 15. Possible pathway of photocatalytic degradation of dyes

In this present case, the photocatalyst, cobalt oxide $\left(\mathrm{Co}_{3} \mathrm{O}_{4}\right)$ nanoparticle has a complex spinel structure in which $\mathrm{Co}^{2+}$ ions occupy at tetrahedral sites and $\mathrm{Co}^{3+}$ ions occupy at octahedral sites of the cubic close-packed lattice of oxide anions ${ }^{[52]}$. The valance band has a strong $\mathrm{O}^{2-}(2 \mathrm{p})$ character while the main contribution of carriers to the conduction band comes from $\mathrm{Co}^{2+} 3 \mathrm{~d}$ orbital ${ }^{[53]}$. Therefore, the charge transfer band $\left(\mathrm{O}^{2-}\right.$ to $\left.\mathrm{Co}^{2+}\right)$ appeared in the wavelength range $300-700 \mathrm{~nm}$ is the key controlling factor for the CoONPs based photocatalytic reactions ${ }^{[23,24]}$. In our case, the charge transfer band at $\lambda_{\max }=$ $330 \mathrm{~nm}$ with a tail in the visible region could be $\mathrm{O}^{2-}$ to $\mathrm{Co}^{2+}$ charge transfer responsible for the generation of key entities electron-hole pairs that force to degrade the dyes. Herein, photocatalytic degradation takes place in presence of visible light source and both the dyes MX and TY have strong absorption band of $\lambda_{\max }=522 \mathrm{~nm}$ and $\lambda_{\max }=409 \mathrm{~nm}$ respectively. Therefore, both the photocatalytic processes and the photosensitized process would work concurrently, although the latter is the predominant process, which could be concluding from the reactivity difference between MX and TY (Figure 12, 14). 


\section{Conclusions}

In this present study, cobalt oxide nanoparticles were successfully synthesized by thermal decomposition of a tetracobalt-Schiff base complex. Analysis of electron microscopy (FE-SEM and HR-TEM) images delineate the existence of cauliflower structured CoONPs with an average size $\sim 80 \mathrm{~nm}$. DLS and zeta potential value, $+33.2 \mathrm{mV}$ attributes the stability of CoONPs in solution phase. The photo-catalytic behavior of CoONPs has been tested against murexide and titan yellow dyes in aqueous phase which ensure more than $90.0 \%$ degradation efficiency for MX and $51.0 \%$ degradation efficacy for TY under visible light irradiation for 40 mins. From the theoretical background and experimental outcomes, it is supposed that the synthetic CoONPs behave as a photosensitizer in the course of catalytic degradation of MX and TY in presence of visible light. Structural differentiation of MX and TY accounts on the different rate of photosensitization of the CoONPs photocatalyst and leading to variation of degradation efficiency towards MX and TY under visible light irradiation. More experimental results need to be performed to understand deeply the photocatalytic role of synthetic CoONPs towards the degradation of MX and TY under visible light exposure. Although the degradation of the organic dyes using the synthetic CoONPs has been examined in laboratory scale, however further efforts need to be carried out to improve the photocatalytic function of the synthetic eco-friendly material focusing on band gap energy, cost-effectiveness and recyclability in industrial scale under various condition.

\section{Acknowledgement}

The work is supported financially by Science and Engineering Research Board (SERB), New Delhi, India under Teachers' Associateship for Research Excellence (NO. TARE/2018 / 000473 dtd. 01/03/2019).

\section{References}

[1] Qian M-D, Sun Y-L, Hu Z-Y, et al. Fluorescent chemo-sensors based on "dually smart" optical micro/nanowaveguides lithographically fabricated with AIE composite resins. Mater. Horiz. 2020; 7: 1782-1789.

[2] Jain A, Singh SK, Arya SK, et al. Protein nanoparticles: Promising platforms for drug delivery applications. ACS Biomater Sci Eng. 2018; 4(12): 3939-3961.

[3] Fan N, Liu H, Zhou Q, et al. Memory devices based on functionalized copolymers exhibiting a linear dependence of switch threshold voltage with the pendant nitro-azobenzene moiety content change. J Mater Chem. 2012; 22: 1995719963.

[4] Sanchez C, Belleville P, Popall M, et al. Applications of advanced hybrid organic-inorganic nanomaterials: From laboratory to market. ChemSoc Rev. 2011; 40: 696-753.

[5] Chang C-M, Hsu C-H, Liu Y-W, et al. Interface engineering: Broad band light and low temperature gas detection abilities using a nano-heterojunction device. Nanoscale. 2015; 7: 20126-20131.

[6] Hou G, Zhang H, Xie G, et al. Ultratrace detection of glucose with enzyme-functionalized single nanochannels. $J$ Mater Chem A. 2014; 2: 19131-19135.

[7] Tong S, Quinto CA, Zhang L, et al. Size-dependent heating of magnetic iron oxide nanoparticles. ACS Nano. 2017; 11(7): 6808-6816.

[8] Wu W, Jiang CZ, Roy VAL. Designed synthesis and surface engineering strategies of magnetic iron oxide nanoparticles for biomedical applications. Nanoscale. 2016; 8: 19421-19474.

[9] Sun M-H, Huang S-Z, Chen L-H, et al. Applications of hierarchically structured porous materials from energy storage and conversion, catalysis, photocatalysis, adsorption, separation, and sensing to biomedicine. Chem Soc Rev. 2016; 45: 3479-3563.

[10] Naha PC, Liu Y, Hwang G, et al. Dextran-coated iron oxide nanoparticles as biomimetic catalysts for localized and pH-activated biofilm disruption. ACS Nano. 2019; 13: 4960 - 4971.

[11] Chen Z, Yin J-J, Zhou Y-T, et al. Dual enzyme-like activities of iron oxide nanoparticles and their implication for diminishing cytotoxicity. ACS Nano. 2012; 6: 4001-4012.

[12] Laurent S, Forge D, Port M, et al. Magnetic iron oxide nanoparticles: Synthesis, stabilization, vectorization, physicochemical characterizations, and biological applications. Chem Rev. 2008; 108: 2064-2110.

[13] Lartigue L, Wilhelm C, Servais J, et al. Nanomagnetic sensing of blood plasma protein interactions with iron oxide nanoparticles: Impact on macrophage uptake. ACS Nano. 2012; 6: 2665-2678.

[14] Long N V, Teranishi T, Yang Y, et al. Iron oxide nanoparticles for next generation gas sensors. Int J Metall Mater Eng. 2015; $1: 119$.

[15] Kim D, Lee N, Park Y, et al. Recent development of nanoparticles for molecular imaging. Bioconjugate Chem. 2017; 
28: 115-123.

[16] Yu S, Ng VMH, Wang F, et al. Synthesis and application of iron-based nanomaterials as anodes of lithium-ion batteries and supercapacitors. J Mater Chem A. 2018; 6: 9332-9367.

[17] Koo B, Xiong H, Slater MD, et al. Hollow iron oxide nanoparticles for application in lithium ion batteries. Nano Lett. 2012; 12: 2429-2435.

[18] Ma Q, Izu N, Masuda Y. Ceria polymer hybrid nanoparticles and assembled films for coating applications. ACS Appl. Nano Mater. 2018; 1: 2112-2119.

[19] Stella C, Soundararajan N, Ramachandran K. Chunk shaped $\mathrm{ZnO} / \mathrm{Co}_{3} \mathrm{O}_{4}$ nanocomposite for ethanol sensor. Adv Mater Lett. 2016; 7: 652-658.

[20] Yoo DH, Cuong TV, Luan VH, et al. Photocatalytic performance of a Ag/ZnO/CCG multidimensional heterostructure prepared by a solution-based method. J Phys Chem C. 2012; 112: 7180-7184.

[21] Sarkar D, Ghosh CK, Mukherjee S, et al. Three dimensional $\mathrm{Ag}_{2} \mathrm{O} / \mathrm{TiO}_{2}$ type-II (p-n) nanoheterojunctions for superior photocatalytic activity. ACS Appl Mater Inter-faces. 2013; 5(2): 331-337.

[22] Wang X, Zhang L. Kinetic study of hydroxyl radical formation in a continuous hydroxyl generation system. $R S C A d v$. 2018; 8: 40632-40638.

[23] Dhas CR, Venkatesh R, Jothivenkatachalam K, et al. Visible light driven photocatalytic degradation of Rhodamine B and Direct Red using cobalt oxide nanoparticles. Ceram Int. 2015; 41: 9301-9313.

[24] Dey D, Kaur G, Patra M, et al. A perfectly linear trinuclear zinc-Schiff base complex: Synthesis, luminescence property and photocatalytic activity of zinc oxide nanoparticle. Inorg Chim Acta. 2014; 421: 335-341.

[25] Rauf MA, Meetani MA, Hisaindee S. An overview on the photocatalytic degradation of azo dyes in the presence of $\mathrm{TiO}_{2}$ doped with selective transition metals. Desalination. 2011; 276: 13-27.

[26] Dey D, Das S, Patra M, et al. Synthesis and characterization of a flower-structured ferromagnetic nickel oxide nanoparticle: Investigation of photocatalytic activity. J Org Inorg Chem. 2015; 1: 1-8.

[27] Tana C, Zhua G, Hojamberdievb M, et al. $\mathrm{Co}_{3} \mathrm{O}_{4}$ nanoparticles-loaded BiOCl nanoplates with the dominant $\{001\}$ facets: Efficient photodegradation of organic dyes under visible light. Appl Catal B: Environ. 2014; 152(153): 425436.

[28] Kumar MRA, Abebe B, Nagaswarupa HP, et al. Enhanced photocatalytic and electrochemical performance of TiO ${ }_{2}$ $\mathrm{Fe}_{2} \mathrm{O}_{3}$ nanocomposite: Its applications in dye decolorization and as supercapacitors. Sci Rep. 2020; $10: 1249$.

[29] Warang T, Patel N, Fernandes R, et al. $\mathrm{Co}_{3} \mathrm{O}_{4}$ nanoparticles assembled coatings synthesized by different techniques for photo-degradation of methylene blue dye. Appl Catal B: Environ. 2013; 132(133): 204-211.

[30] Das S, Sahu A, Joshi M, et al. Ligand centered radical activity by a zinc-Schiff base complex towards catechol oxidation. Chemistry Select. 2018; 3: 10774-10781.

[31] De A, Dey D, Pal CK, et al. Carbinol mediated clusterization of Nickel(II) ions in a Schiff base backbone: Structural \& solution properties, phosphoester cleavage activity including theoretical support. J Mol Struct. 2019; 1195: 293-301.

[32] Garai M, Das A, Joshi M, et al. Synthesis and spectroscopic characterization of a photo-stable tetrazinc(II)-Schiff base cluster: A rare case of ligand centric phenoxazinone synthase activity. Polyhedron. 2018; 156: 223-230.

[33] Pal S, Chowdhury B, Patra M, et al. Ligand centered radical pathway in catechol oxidase activity with a trinuclear zinc-based model: Synthesis, structural characterization and luminescence properties. Spectrochim Acta Part A: Mol Biomol Spectros. 2015; 144: 148-154.

[34] CrysAlisPro 1.171.39.35c. Rigaku Oxford Diffraction. Tokyo, Japan: Rigaku Corporation; 2017.

[35] Sheldrick GM. SHELXT-Integrated space-group and crystal-structure determination. Acta Crystallogr A Found Adv. 2015; 71(1): 3-8.

[36] Sheldrick GM. Crystal structure refinement with SHELXL. Acta Crystallogr C Struct Chem. 2015; 71: 3-8.

[37] Dolomanov OV, Bourhis LJ, Gildea RJ, et al. OLEX2: A complete structure solution, refinement and analysis program. J Appl Cryst. 2009; 42: 339-341.

[38] Jiang L, Zhang D-Y, Suo J-J, et al. Synthesis, magnetism and spectral studies of six defective dicubane tetranuclear $\left\{\mathrm{M}_{4} \mathrm{O}_{6}\right\}\left(\mathrm{M}=\mathrm{Ni}^{\mathrm{II}}, \mathrm{Co}^{\mathrm{II}}, \mathrm{Zn}^{\mathrm{II}}\right)$ and three trinuclear $\mathrm{Cd}^{\mathrm{II}}$ complexes with polydentate Schiff base ligands. Dalton Trans. 2016; 45: 10233-10248.

[39] Jacobs C, Muller RH. Production and characterization of a budesonide nanosuspension for pulmonary administration. Pharm Res. 2002; 19: 189-194.

[40] Hitkari G, Sandhya S, Gajanan P, et al. Synthesis of chromium doped cobalt oxide $\left(\mathrm{Cr}: \mathrm{Co}_{3} \mathrm{O}_{4}\right)$ nanoparticles by coprecipitation method and enhanced photocatalytic properties in the visible region. J Mater Sci Eng. 2018; 7: 419.

[41] Jassal V, Shanker U, Kaith BS, et al. Green synthesis of potassium zinc hexacyanoferrate nanocubes and their potential application in photocatalytic degradation of organic dyes. RSC Adv. 2015; 5(33): 26141-26149.

[42] Adekunle AS, Oyekunle JAO, Durosinmi LM, et al. Potential of cobalt and cobalt oxide nanoparticles as nanocatalyst towards dyes degradation in wastewater. Nano-Structures \& Nano-Objects. 2010; 21: 100405. 
[43] Kasiri MB, Aleboyeh H. Modeling and optimization of heterogeneous photo-Fenton process with response surface methodology and artificial neural networks. Environ. Sci. Technol. 2008; 42(21): 7970-7975.

[44] Hendekhale NR, Mohammad-Khaha A. A novel synthesis of $\mathrm{Co}_{2} \mathrm{ZrO}_{5}$ and $\mathrm{m}-\mathrm{ZrO}_{2}$ nanoparticles by sono-precipitation and hydrothermal methods and their application in UV/Visible-photocatalytic studies. J Environ Chem Eng. 2020; 8(5): 104065.

[45] Pal S, Mondal S, Maity J, et al. Synthesis and characterization of $\mathrm{ZnO}$ nanoparticles using moringa oleifera leaf extract: Investigation of photocatalytic and antibacterial activity. Int J Nanosci Nanotechnol. 2018; 14: 111-119.

[46] Hiremath S, Antony Raj MAL, Chandra Prabha MN, et al. Tamarindusindica mediated biosynthesis of nano $\mathrm{TiO}_{2}$ and its application in photocatalytic degradation of Titan yellow. J Environ Chem Eng. 2018; 6(6): 7338-7346. Available from: https://doi.org/10.1016/j.jece.2018.08.052.

[47] Girish KM, Prashantha SC, Nagabhushana H, et al. Multi-functional $\mathrm{Zn}_{2} \mathrm{TiO}_{4}$ : $\mathrm{Sm}^{3+}$ nanopowders: Excellent performanceas an electrochemical sensor and an UV photocatalyst. $J$ Sci: Adv Mat Dev. 2018; 3: 151-160.

[48] Vidya C, Manjunatha C, Sudeep M, et al. Photo-assisted mineralisation of titan yellow dye using ZnO nanorods synthesised via environmental benign route. SN Appl Sci. 2020; 2: 743.

[49] Tana C, Zhua G, Hojamberdievb M, et al. $\mathrm{Co}_{3} \mathrm{O}_{4}$ nanoparticles-loaded $\mathrm{BiOCl}$ nanoplates with the dominant $\{001\}$ facets: Efficient photodegradation of organic dyes under visible light. Appl Catal B: Environ. 2014; 152-153: 425-436.

[50] Anwer H, Mahmood A, Lee J, et al. Photocatalysts for degradation of dyes in industrial effluents: Opportunities and challenges. Nano Research. 2019; 12(5): 955-972.

[51] $\mathrm{Fu} \mathrm{H}$, Pan C, Yao W, et al. Visible-light-induced degradation of rhodamine B by nanosized $\mathrm{Bi}_{2} \mathrm{WO}_{6}$. J Phys Chem B. 2005; 109: 22432-22439.

[52] Miedzinska KME, Hollebone BR. An assigment of the optical absorption spectrum of mixed valence $\mathrm{Co}_{3} \mathrm{O}_{4}$ spinel films. J Phys Chem Solids. 1987; 48: 649-656.

[53] Barreca D, Massignan C, Daolio S, et al. Composition and microstructure of cobalt oxide thin films obtained from a novel cobalt(II) precursor by chemical vapor deposition. Chem Mater. 2001; 13: 588-593. 